\title{
Long-term prognostic value of coronary flow reserve in psoriasis patients
}

\author{
Stefano Piaserico ${ }^{\mathrm{a}, 1}$, Elena Osto ${ }^{\mathrm{b}, \mathrm{c}, 1}$, Giulia Famoso ${ }^{\mathrm{d}}$, Roberta Montisci ${ }^{\mathrm{e}}$, Laura De Michieli ${ }^{\mathrm{d}}$, \\ Irene Zanetti ${ }^{\mathrm{d}}$, Sabino Iliceto ${ }^{\mathrm{d}}$, Francesco Tona ${ }^{\mathrm{d}, *}$ \\ ${ }^{a}$ Dermatology Unit, Department of Medicine, University of Padova, Padova, Italy \\ ${ }^{\mathrm{b}}$ University Heart Center, University of Padova, Padova, Italy \\ ${ }^{\mathrm{c}}$ Institute of Clinical Chemistry, University of Padova, Padova, Italy \\ ${ }^{\mathrm{d}}$ Department of Cardiac, Thoracic, Vascular Sciences and Public Health, University of Padova, Padova, Italy \\ ${ }^{\mathrm{e}}$ Clinical Cardiology, Department of Medical Science and Public Health, University of Cagliari, Cagliari, Italy
}

\section{H I G H L I G H T S}

- This study analyzed the prognostic role of coronary flow reserve in psoriasis.

- 153 psoriatic patients without cardiovascular disease were included.

- Coronary microvascular dysfunction was present in $15 \%$ of patients.

- Coronary flow reserve may be a prognostic marker for events-free survival.

A R T I C L E IN F O

\section{Keywords:}

Psoriasis

Coronary microcirculation

Prognosis

Coronary flow reserve

Cardiovascular risk

\begin{abstract}
A B S T R A C T
Background and aims: Psoriasis affects more than $3 \%$ of the general population and is associated with an increased risk of premature cardiovascular events and death. We assessed the prognostic role of coronary flow reserve (CFR) as a marker of coronary microvascular function in psoriasis patients asymptomatic for cardiovascular disease.

Methods: We retrospectively analyzed 153 prospectively collected patients affected by psoriasis (123 male; age $36 \pm 8$ years) without cardiovascular disease. CFR in the left anterior descending coronary artery was detected by transthoracic Doppler echocardiography, at rest, and during adenosine infusion. CFR was the ratio of hyperemic to resting diastolic flow velocity. CFR $\leq 2.5$ was the cut off to define the presence of coronary microvascular dysfunction (CMD).

Results: CMD was present in 23 patients (15\%). Multivariable logistic regression analysis showed that CMD was associated with severe psoriasis (OR 3.1, $p=0.03$ ), psoriatic arthritis (OR 2.9, $p=0.03$ ), hypertension (OR 4.1, $p=0.009$ ), and time elapsing since psoriasis diagnosis $>6$ years (OR $1.9, p=0.03$ ). Patients with CFR $\leq 2.5$ had a lower survival free from events $(p<0.0001)$.

Conclusions: In psoriasis patients, CFR may be a reliable prognostic marker for cardiovascular event-free survival and may help identify patients at higher risk of developing cardiovascular complications. Whether novel biologic therapies able to reduce skin disease will improve CMD and prognosis in these patients needs to be further studied, prospectively.
\end{abstract}

\section{Introduction}

Patients affected by psoriasis, similarly to other chronic inflammatory systemic immune-mediated diseases, such as rheumatoid arthritis (RA), systemic lupus erythematosus (SLE) and systemic sclerosis (SSc), suffer from increased cardiovascular morbidity and mortality due to premature and accelerated atherosclerosis [1].

Systemic chronic inflammation is regarded as a major causal mechanism leading to atherosclerosis-driven clinical and subclinical coronary artery disease in patients with psoriasis and other immunemediated diseases [2,3].

Recent positive results obtained by monoclonal antibodies against

\footnotetext{
* Corresponding author.

E-mail address: francesco.tona@unipd.it (F. Tona).

${ }^{1}$ These authors contributed equally to the work.
} 
interleukin (IL)-1b in secondary prevention of cardiovascular events in patients with coronary artery disease and residual inflammatory risk, further support inflammation as a therapeutic target in atherosclerosis [4].

Indeed, in patients with severe psoriasis, biologic therapies (i.e. anti TNF $\alpha$, anti-IL-12/23 and anti- IL-17) were associated with favorable modulation of coronary plaque characteristics on the short term and anti TNF $\alpha$ decreased risk for myocardial infarction over a longer period of treatment $[5,6]$.

Coronary microvascular dysfunction (CMD) describes a disease entity common to various clinical situations in which a reduced coronary flow reserve (CFR) (i.e. the ratio between blood flow during maximal coronary vasodilation and resting flow) along with evidence of myocardial ischaemia are not secondary to epicardial coronary artery stenosis [7]. We previously reported in young patients with psoriasis, without traditional cardiovascular risk factors or angiographic evidence for coronary artery disease, higher prevalence of CMD detected by CFR measured by transthoracic Doppler echocardiography (TDE). In these patients, the degree of CMD was directly correlated to the disease severity evaluated by the Psoriasis Area Severity Index (PASI) [8] and CFR was improved by anti-TNF $\alpha$ therapy, which is commonly considered as the first line biologic agent for the treatment of psoriasis [9].

Emerging data documents that CMD, despite the absence of obstructive CAD, is not a benign condition. Indeed, in patients with systemic immune-mediated diseases like SSc, CMD is associated to higher risk of adverse cardiovascular outcomes [10].

The starting hypothesis of our work is that even in patients with psoriasis the CMD may play a prognostic role. Consequently, the aim and the novelty of the present study was to characterize in patients with moderate-to-severe psoriasis the cardiovascular long-term prognostic value of impaired CFR detected by TDE, as a cost-effective tool to riskstratify young patients with CMD without hemodynamically significant stenosis as detected by coronary multi-slice computed tomography [MSCT].

\section{Materials and methods}

This study is a retrospective analysis of prospectively collected data at a single center. We enrolled 153 consecutive patients affected by moderate-to-severe psoriasis (123 male; mean age, $36 \pm 8$ years, range 18-50), recruited between January 2009 and December 2015. All patients had a diagnosis of moderate to severe psoriasis since at least 12 months, and stable plaque psoriasis since at least 2 months before screening. Patients were included if they met the following inclusion criteria: available CFR and MSCT evaluation, age $<50$ years, no symptoms or history of cardiovascular disease. The following risk factors for coronary artery disease were assessed at the time of CFR evaluation: hypertension, diabetes mellitus, hypercholesterolemia, smoking, high-sensitive C-reactive protein (hsCRP), family history of heart disease, body mass index (BMI). For risk factors definition see Supplementary Material online. Exclusion criteria were high blood pressure at CFR evaluation, history or clinical evidence of renal (serum creatinine $>133 \mu \mathrm{mol} / \mathrm{L}$ in men and $>120 \mu \mathrm{mol} / \mathrm{L}$ in women), or hepatic disease and malignant or infectious disease. The activity of psoriasis was determined using the validated disease-specific activity index PASI.

\subsection{Psoriasis Area Severity Index (PASI) evaluation}

The body is divided into four regions comprising the head, upper extremities, trunk, and lower extremities. In each of these areas, the fraction of total surface area affected is graded on a $0-6$ scale ( 0 for no involvement up to 6 for greater than $90 \%$ involvement). The various body regions are weighted to reflect their respective proportion of body surface area. The composite PASI score is then calculated by multiplying the sum of the individual-severity scores for each region by the weighted area-of-involvement score for that respective region, and then summing the four resulting quantities. The highest potential PASI score is 72; the lowest is 0. PASI scores are nearly continuous, with 0.1 increments within these values. PASI 5-10 defined moderate psoriasis, PASI $>10$ defined a severe psoriasis.

\subsection{Echocardiography methods and coronary flow velocity reserve assessment}

Transthoracic Doppler echocardiography was performed with a commercially available ultrasound system (Vivid 7, GE Medical System, Inc., Hortem, Norway). Doppler echocardiography and CFR assessment has been previously detailed [11-14]. A CFR $\leq 2.5$ was considered abnormal [15], and the population was dichotomized according to this cutoff.

\subsection{MSCT coronary angiography protocol and interpretation}

Patients with abnormal CFR underwent to MSCT coronary angiography to exclude epicardial significant CAD and to measure calcium score. Coronary MSCT was performed using a 64-slice dual-source scanner (Definition, Siemens Medical System, Forchheim, Germany). MSCT protocol and interpretation has been previously detailed [16].

\subsection{Adverse outcomes during follow-up}

Subjects were followed prospectively to a combined endpoint of major adverse cardiovascular events (MACE). During a follow up performed every 3 months, records of patients reporting an event were reviewed by an events committee blinded to CFR evaluation and tabulated as death, nonfatal myocardial infarction (MI), nonfatal stroke; and hospital stay for congestive heart failure (CHF), angina, and other vascular events. Those with death, nonfatal MI, nonfatal stroke, or CHF hospital stay were categorized as having MACE. Angina and myocardial infarctions were defined by ESC guidelines $[17,18]$.

\subsection{Statistical analysis}

Continuous variables with no/mild skew were presented as mean $\pm \mathrm{SD}$; skewed measures were represented as median with first and third quartiles (Q1-Q3). Discrete variables were summarized as frequencies and percentages. The distribution of the data was analyzed with a 1-sample Kolmogorov-Smirnov test. Categorical variables were compared by the $\chi^{2}$ test or the Fisher exact test as appropriate. Continuous data were compared by use of the 2-tailed paired or unpaired $t$-test (for normally distributed data sets) or the Mann-Whitney $U$ or Wilcoxon signed-rank test (for skewed variables). Stepwise logistic regression analysis was used to model normal versus abnormal CFR $(\leq 2.5)$ and risk factors. Baseline characteristics were chosen for entry into multivariable models on the basis of their discrimination between normal and abnormal CFR and on unadjusted association with CFR $\leq 2.5$ of $p \leq 0.1$. In order to account for possible modulator effects, we then forced all other variables from Table 1 into the model, one at a time. A combination of forward and backward selection procedures was used to aid in determining the best model of factors independently associated with $\mathrm{CFR} \leq 2.5$. Median values for age, severity of psoriasis and duration of psoriasis were selected as a cut point for simplicity in the multivariable analysis. Summary statistics for the regression models included the $c$-statistic (a measure of association of predicted probabilities and observed prevalence of a binary outcome) and $\mathrm{R}^{2}$ (rescaled for use in logistic regression). As for the retrospective follow-up, stepwise logistic regression analysis was also used to model event versus non event (as dependent variable) and CMD. Event-free survival curves were traced by use of the Kaplan-Meier method and compared by log-rank test. All tests were two-sided and statistical significance was accepted if the null hypothesis could be rejected at $p<0.05$. Data 
Table 1

Clinical characteristics of the study population, stratified by the presence or absence of coronary microvascular dysfunction (CMD; CFR $\leq 2.5$ ).

\begin{tabular}{|c|c|c|c|c|}
\hline & All patients $(\mathrm{n}=153)$ & $\begin{array}{l}\text { CMD present (CFR } 2.0 \pm 0.4 \text {, range } 1.2-2.5 \text {; } \\
\mathrm{n}=23 \text { ) }\end{array}$ & $\begin{array}{l}\text { CMD absent }(\text { CFR } 3.3 \pm 0.6 \text {, range } 2.6-6.3 \text {; } \\
\mathrm{n}=130)\end{array}$ & $p$ \\
\hline Age, years & $36 \pm 8$ & $40 \pm 9$ & $36 \pm 7$ & 0.03 \\
\hline Males, n (\%) & $122(79.7)$ & $20(87)$ & $102(78.5)$ & 0.5 \\
\hline PASI & $15(11-20)$ & $18(11-20)$ & $13(11-15)$ & 0.02 \\
\hline Time elapsing since psoriasis diagnosis, years & $16(10-22)$ & $17(10-26)$ & $14(9-21)$ & 0.03 \\
\hline Presence of PsA, n (\%) & $50(32.6)$ & $11(47.8)$ & $39(30)$ & 0.03 \\
\hline $\mathrm{BMI}, \mathrm{Kg} / \mathrm{m}^{2}$ & $26.39 \pm 4$ & $25.76 \pm 3.91$ & $26.48 \pm 4.01$ & 0.4 \\
\hline Hypertension, $\mathrm{n}(\%)$ & $24(15.8)$ & $9(39.1)$ & $15(11.6)$ & 0.003 \\
\hline Family history of heart disease, $\mathrm{n}(\%)$ & $36(23.5)$ & $10(43.4)$ & $26(20)$ & 0.02 \\
\hline Current smoking, n (\%) & $52(33.9)$ & $8(34.8)$ & $44(33.8)$ & 0.6 \\
\hline Previous smoking, n (\%) & $43(28.1)$ & $5(21.7)$ & $38(29.2)$ & 0.7 \\
\hline $\mathrm{SBP}, \mathrm{mmHg}$ & $121 \pm 14$ & $124 \pm 15$ & $121 \pm 14$ & 0.5 \\
\hline $\mathrm{DBP}, \mathrm{mmHg}$ & $76 \pm 11$ & $75 \pm 7$ & $76 \pm 12$ & 0.6 \\
\hline Total cholesterol, $\mathrm{mg} / \mathrm{dL}$ & $194.74 \pm 43.17$ & $203.56 \pm 42.43$ & $193.31 \pm 43.31$ & 0.3 \\
\hline Triglycerides, mg/dL & $110.53 \pm 61.96$ & $113.50 \pm 80.61$ & $110.05 \pm 58.80$ & 0.8 \\
\hline hsCRP, mg/L & $2.39 \pm 3.26$ & $3.24 \pm 1.81$ & $2.31 \pm 3.37$ & 0.4 \\
\hline
\end{tabular}

BMI, Body Mass Index; CFR, coronary flow reserve; CMD, coronary microvascular dysfunction; hsCRP, high sensitive-C reactive protein; PASI, Psoriasis Area Severity Index; PsA, psoriatic arthritis. DBP, diastolic blood pressure; SBP, systolic blood pressure.

Data are presented as mean \pm SD.

were analyzed with SPSS software version 24.0 (Chicago, SPSS, Inc., Chicago, Illinois). The authors had full access to the data and take full responsibility for its integrity. All authors have read and agree to the manuscript as written. The study protocol was reviewed and approved by the institutional ethical committee. All participants gave their written informed consent to participate in the study.

\subsection{Sample size determination}

Although the study sample size was limited, a priori power calculations based on a one tailed $t$-test to obtain a power of 0.80 , a significance level of 0.05 and an effect size based on published values of standard deviations and MACE incidence in moderate-to-severe psoriasis, would estimate that with 1:1 matching of patients without CMD and patients with CMD and an assumed relative survival free from MACE of 0.8 in the group with CMD, we would need approximately 34 patients in each group to show a difference.

A post-hoc power analysis (assuming $\alpha=0.05$ ) estimated that with 130 patients without CMD and 23 patients with CMD, with an event incidence of $0 \%$ in patients without CMD and $21 \%$ in patients with CMD, we reject the null hypothesis of equal survival with $94.1 \%$ power.

\section{Results}

\subsection{Baseline characteristics}

There were no adverse reactions to adenosine. The baseline characteristics of the 153 patients are summarized in Table 1 . The mean duration of follow-up was $5.53 \pm 2.42$ years (range, 0.6-9.4 years). The study population included $24(15.7 \%)$ subjects with hypertension, $57(37.2 \%)$ with hypercholesterolemia, $52(33.9 \%)$ current cigarette smokers and $36(23.5 \%)$ with a family history of heart disease. No patient was diabetic. Mean BMI was $26.3 \pm 4$. Total cholesterol was $194 \pm 43 \mathrm{mg} / \mathrm{dL}$, and triglycerides were $110 \pm 61 \mathrm{mg} / \mathrm{dL}$. In the whole study group CFR was $3.16 \pm 0.79$. On individual patient analysis, CFR was $>2.5$ in 130 patients (84.9\%), and $\leq 2.5$ in 23 patients (15.1\%). The presence of epicardial artery stenosis was excluded by MSCT in all of 23 patients with CFR $\leq 2.5$ and Calcium Score was $<10$ in all patients with $\mathrm{CFR} \leq 2.5$. For hemodynamic parameters during CFR evaluation see the Supplementary results online.

\subsection{Characteristics of patients with $C M D(C F R \leq 2.5)$}

Patients with CMD were older ( $40 \pm 9$ vs $37 \pm 7$ years, $p=0.03$ ), had a greater severity of psoriasis (PASI 18 [11-20] vs 13 [11-15], $p=0.02$ ), a longer time elapsing since psoriasis diagnosis (17 [10-26] vs 14 [9-21] years, $p=0.03$ ), and more frequently had psoriatic arthritis $(52.4 \%$ vs $29.3 \%, p=0.03$ ), hypertension $(38.1 \%$ vs $12.0 \%$, $p=0.003)$ and family history of heart disease $(42.9 \%$ vs $20.3 \%$, $p=0.024)$ (Table 1).

\subsection{Factors associated with $C M D(C F R \leq 2.5)$}

In unadjusted logistic regression analysis, the significant or marginally significant $(p \leq 0.1)$ risk factors were age $(p=0.03)$, time elapsing since psoriasis diagnosis $>6$ years $(p=0.03)$, hypertension $(p=0.002)$, PASI $>10(p=0.09)$, and PsA $(p=0.1)$. Factors independently associated with CMD are summarized in Table 2 . When modelled using a stepwise regression and adjusting for baseline differences and predictors at unadjusted logistic regression analysis, the factors independently associated with CMD were time elapsing since psoriasis diagnosis $>6$ years and hypertension $(p=0.03$ and $p=0.007$, respectively) (Table 2 ). The inclusion of heart rate-pressure product did not greatly affect the model and no other clinical characteristic entered as a significant covariate. To exclude the modulating effect of other variables, we also added variables marginally significant in unadjusted analysis (model 2). In this model, PASI $>10$ and PsA were also independently associated with CMD, but their addition to the model did not significantly affect the relationship between CMD and hypertension or time elapsing since psoriasis diagnosis $>6$ years (Table 2). When other conditions (current smoking, BMI, and total cholesterol) were forced into the model (potentially overfitting it), time elapsing since psoriasis diagnosis $>6$ years and hypertension remained significantly associated with $\operatorname{CMD}(p=0.03$ and $p=0.02$, respectively) (model 3).

\subsection{Major adverse cardiac events}

A total of 5 patients experienced a MACE. All of them had a CFR $\leq 2.5$ at the beginning of the study. Three patients experienced a type 1 myocardial infarction. One vessel critical epicardial stenosis was diagnosed in 2 patients, which were treated with percutaneous revascularization. Three vessels critical epicardial stenosis was diagnosed in only one patient treated with surgical revascularizazion. Epicardial 
Table 2

Factors independently associated with coronary microvascular dysfunction (CFR $\leq 2.5$ ).

\begin{tabular}{|c|c|c|c|}
\hline & OR & $95 \% \mathrm{CI}$ & $p$ \\
\hline \multicolumn{4}{|c|}{ Model 1: obtained by stepwise logistic regression (C-statistic $=0.80 ; \mathrm{R}^{2}=0.62$ ) } \\
\hline Age & 1 & $0.9-1.1$ & 0.6 \\
\hline Time elapsing since psoriasis diagnosis $>6$ years & 2.02 & $1.4-5.4$ & 0.03 \\
\hline Hypertension & 4.4 & $1.4-13.4$ & 0.007 \\
\hline \multicolumn{4}{|c|}{ Model 2: obtained also including variables marginally significant $(p \leq 0.1)\left(C\right.$-statistic $\left.=0.78 ; R^{2}=0.61\right)$} \\
\hline Age & 1 & $0.8-1.09$ & 0.8 \\
\hline Time elapsing since psoriasis diagnosis $>6$ years & 1.9 & $1.2-4.6$ & 0.03 \\
\hline Hypertension & 4.1 & $1.3-12.1$ & 0.009 \\
\hline PASI $>10$ & 3.1 & $1.1-8.7$ & 0.03 \\
\hline PsA & 2.9 & $1.07-8.1$ & 0.03 \\
\hline \multicolumn{4}{|c|}{ Model 3: obtained forcing other variables $\left(C\right.$-statistic $=0.87 ; \mathrm{R}^{2}=0.70$ ) } \\
\hline Age & 1 & $0.8-1.06$ & 0.7 \\
\hline Time elapsing since psoriasis diagnosis $>6$ years & 1.7 & $1.2-3.8$ & 0.03 \\
\hline Hypertension & 3.6 & $1.2-9.1$ & 0.02 \\
\hline PASI $>10$ & 1.8 & $1.3-6.2$ & 0.04 \\
\hline PsA & 1.8 & $1.04-6.1$ & 0.07 \\
\hline
\end{tabular}

CI, confidence interval; OR, odds ratio.

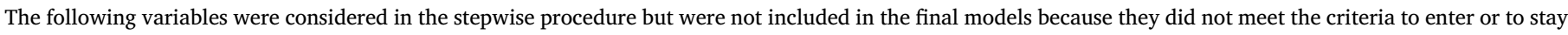
in the model: male gender, hsCRP.

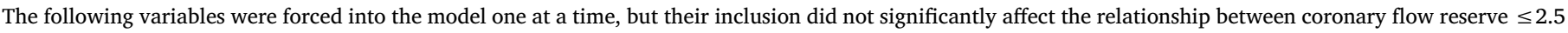
and variables of model 1: current smoking, BMI, and total cholesterol.

coronary arteries were normal in the other 2 patients with MINOCA (myocardial infarction with non-obstructive coronary arteries) (Table 3) [19].

The baseline features of patients with and without cardiovascular events are shown in Table 4. Patients with cardiovascular events were older $(p=0.04)$, had an higer PASI $(p=0.001)$, longer time elapsing since psoriasis diagnosis $(p=0.04)$, and higher prevalence of hypertension $(p=0.002)$. Cardiological therapy and specific therapy for psoriasis were comparable in the two groups. Hyperemic (during adenosine infusion) diastolic coronary flow and CFR were lower in patients with MACE $(57$ [48-61] vs 70 [60-80] cm/s, $2.1 \pm 0.2$ vs $3.1 \pm 0.7$; $p=0.03$ and $p=0.001$, respectively). CMD was present in $18(12.2 \%)$ patients without MACE and in all $5(100 \%)$ patients with MACE $(p<0.0001)$. Fig. 1 shows the cumulative survival free from cardiovascular events according to the presence of CMD. The prevalence of hypertension was the only difference between patients with CMD and MACE and patients with CMD without MACE ( $80 \%$ vs 27.8\%, $p=0.03$ ). PASI tended to be higher in patients with CMD and MACE compared with patients with CMD without MACE $(p=0.06)$. In unadjusted logistic regression analysis, CMD was a significant risk factor for MACE (OR 9.9 (95\% CI 6.1-15.8), $p<0.0001$. Univariable prediction of cardiovascular events is presented in Table 5.

\section{Discussion}

In this study, we found CFR to be reduced in $15 \%$ of asymptomatic psoriasis patients without evidence of epicardial coronary stenosis. Of note, our study patients were relatively young (mean age 36 years) and with a rather low prevalence of traditional CV risk factors. This is in agreement with previous studies that reported abnormal CFR in patients with psoriasis or other systemic inflammatory diseases (such as SLE, ankylosing spondylitis, RA, inflammatory bowel disease) with normal coronary arteries, indicating that microcirculation may be affected before the occurrence of coronary atherosclerosis $[1,8]$.

Most importantly, CFR was found to predict MACE-free survival in our psoriasis patients. Patients with CMD had significantly worse outcome than patients without CMD. Twenty one percent of psoriasis patients with an impaired CFR developed a MACE. This is an impressive outcome considering the age of our study patients, ranging from 18 to 50 years.

Patients affected by severe psoriasis show increased risk of premature CV events and death [20-22]. Traditional CV risk factors appear to contribute to cardiovascular events development in patients with severe psoriasis. However, the increased risk noted in this common inflammatory condition is not completely captured by traditional risk assessment, and persists beyond adjustment for these factors. The analysis of the General Practice Research Database showed that patients with severe psoriasis had a 53\% [23] and 57\% [20] increased risk for

Table 3

Demographic and clinical characteristics of patients with events.

\begin{tabular}{|c|c|c|c|c|c|c|c|c|}
\hline No. & $\begin{array}{l}\text { Age/ } \\
\text { gender }\end{array}$ & Time from diagnosis (years) & Type of MI & Psoriasis treatment & Angiographic CAD & Revascularization therapy & Time from CFR (months) & CFR \\
\hline 1 & $44 / \mathrm{M}$ & 26 & MINOCA $^{\mathrm{a}}$ & Infliximab & none & / & 5 & 2.3 \\
\hline 2 & $38 / \mathrm{M}$ & 7 & Type $1 \mathrm{MI}^{\mathrm{b}}$ & Acitretin & $70 \% \mathrm{LAD}$ & PTCA + DES & 4 & 1.8 \\
\hline 3 & $45 / \mathrm{M}$ & 6 & MINOCA $^{\mathrm{a}}$ & Ustekinumab & none & / & 77 & 2.0 \\
\hline 4 & $53 / \mathrm{M}$ & 39 & Type $1 \mathrm{MI}^{\mathrm{b}}$ & Etanercept & $90 \%$ RCA & PTCA + DES & 2 & 2.2 \\
\hline 5 & $46 / \mathrm{M}$ & 23 & Type $1 \mathrm{MI}^{\mathrm{b}}$ & Adalimumab & TCO Cx, 80\% LAD, 70\% PDA & CABG & 100 & 2.3 \\
\hline
\end{tabular}

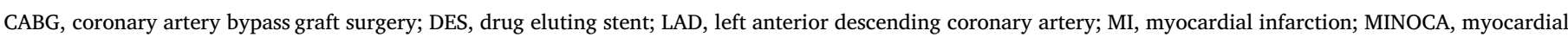

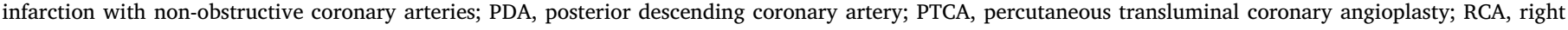
coronary artery; TCO total coronary occlusion. Other abbreviations as in Table 1.

a MINOCA: MI with no angiographic obstructive coronary artery disease ( $\geq 50 \%$ diameter stenosis in a major epicardial vessel).

b Type 1 myocardial infarction: MI caused by atherothrombotic coronary artery disease and usually precipitated by atherosclerotic plaque disruption (rupture or erosion). 
Table 4

Clinical characteristics of patients with and without cardiovascular events.

\begin{tabular}{|c|c|c|c|}
\hline & MACE $(n=5)$ & $\begin{array}{l}\text { No MACE } \\
(\mathrm{n}=148)\end{array}$ & $p$ \\
\hline Age, years & $43 \pm 6$ & $36 \pm 8$ & 0.04 \\
\hline Males, n (\%) & $5(100)$ & $117(79)$ & 0.3 \\
\hline PASI & $15(7-31)$ & $10(3-15)$ & 0.001 \\
\hline Time elapsing since psoriasis diagnosis, years & $13(4-25)$ & $6(3-11)$ & 0.04 \\
\hline Presence of PsA, n (\%) & $2(40)$ & $48(32)$ & 0.4 \\
\hline BMI, $\mathrm{m}^{2} / \mathrm{Kg}$ & $27 \pm 5$ & $26 \pm 4$ & 0.7 \\
\hline Hypertension, $\mathrm{n}(\%)$ & $4(80)$ & $21(14)$ & 0.002 \\
\hline Family history of heart disease, $\mathrm{n}(\%)$ & $0(0)$ & $36(24)$ & 0.2 \\
\hline Current smoking, n (\%) & $0(0)$ & $54(36)$ & 0.1 \\
\hline Previous smoking, $\mathrm{n}(\%)$ & $0(0)$ & $50(33)$ & 0.1 \\
\hline $\mathrm{SBP}, \mathrm{mmHg}$ & $122 \pm 22$ & $121 \pm 14$ & 0.9 \\
\hline $\mathrm{DBP}, \mathrm{mmHg}$ & $77 \pm 12$ & $76 \pm 11$ & 0.8 \\
\hline Total cholesterol, mg/dL & $200 \pm 18$ & $194 \pm 43$ & 0.8 \\
\hline Triglycerides, mg/dL & $94 \pm 65$ & $110 \pm 62$ & 0.7 \\
\hline hsCRP, mg/L & $2.1 \pm 0.2$ & $2.4 \pm 0.7$ & 0.1 \\
\hline $\mathrm{hDFV}, \mathrm{cm} / \mathrm{s}$ & $57(48-61)$ & $70(60-80)$ & 0.03 \\
\hline CFR & $2.1 \pm 0.2$ & $3.1 \pm 0.7$ & 0.001 \\
\hline
\end{tabular}

hDFV, hyperemic diastolic flow velocity. Other abbreviations as in Table 1.

Data are presented as mean \pm SD or as median with first and third quartiles (Q1-Q3).

Log Rank $p<0.0001$

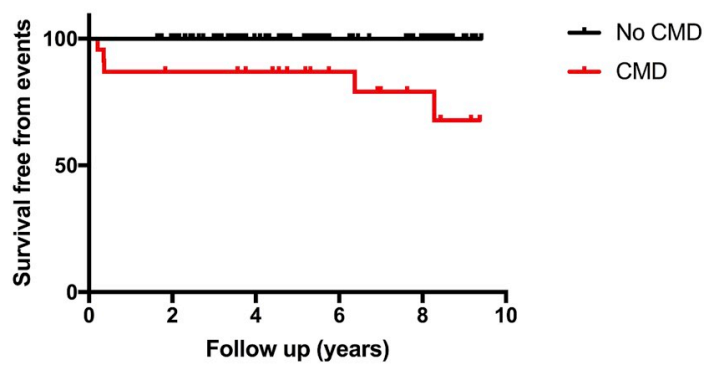

Fig. 1. Kaplan Meier cardiac event-free survival curves: comparison of patients with or without CMD $(p<0.0001)$.

major adverse cardiac events and CV mortality, respectively, after adjusting for age, gender, diabetes, hypertension, tobacco use, and hyperlipidemia.

As inflammation is a critical component of atherosclerosis pathophysiology, chronic inflammation in psoriasis patients may play a significant role in the shared mechanisms driving accelerated-atherosclerosis. During a psoriasis flare, there are more than 1 billion immune cells activated within the body. Psoriasis is characterized by increased pro-inflammatory cytokine levels and activation of myeloid dendritic cells, neutrophils, and Th cells (mostly Th17). T cells secrete intermediates (e.g., IL-17F, IL-17A, and IL-22), which, in turn, activate keratinocytes and further stimulate the production of pro-inflammatory cytokines. IL-17A, IL-1, and TNF- $\alpha$ play also a role in atherosclerosis by driving cell-mediated immunity.

The prognostic value of an impaired CFR has been well demonstrated in several groups of patients, even without coronary artery disease [24-26]. In fact, impaired CFR might not only represent the extent of disease in conductance vessels but might also be related to pure microvascular vasomotor dysfunction, because in the absence of hemodynamically significant stenosis maximal increase of blood flow is predominantly determined by resistance vessels of the coronary microcirculation. Intriguingly, a reduced CFR has been showed to predict a worse cardiovascular prognosis also in asymptomatic patients with diabetes [27] or systemic sclerosis [10]. In our study patients, CFR impairment was not associated to significant coronary artery disease. These findings are suggestive of CMD, which may represent an early marker of accelerated coronary atherosclerosis and contribute to the increased cardiovascular morbidity and mortality in these patients. In keeping with this, our study suggests that CMD may precede, at least in some cases, the onset of an epicardial disease, susceptible of percutaneous or surgical revascularization. Indeed, coronary arteries were normal at MSCT at the time of CFR assessment in all our patients with type 1 myocardial infarction.

Systemic inflammation has been shown to be an important risk factor for CMD, similarly to traditional CV risk factors, such as smoking, diabetes mellitus, hypertension, and hyperlipidemia [1,28-31]. In our population, a blunted CFR was associated with a severe psoriasis and the presence of psoriatic arthritis, both associated with a higher systemic inflammatory burden [32,33]. In this context, a psoriasis/psoriatic arthritis-specific element appears to contribute to the CMD and the increased cardiovascular risk associated with psoriasis, although the precise nature of this remains to be determined. Soluble factors including increased levels of the pro-inflammatory cytokines, TNF- $\alpha$ and IL-17, may be important. TNF- $\alpha$ may impair the activation of endothelial nitric oxide synthase (eNOS), degrade eNOS mRNA, alter vasomotor function acting on vascular smooth muscle cells and induces oxidative stress by increasing the production of reactive oxygen species [34]. Some studies reported that also in healthy individuals circulating levels of several different pro-inflammatory cytokines, in particular IL-6 and TNF- $\alpha$, are associated with risk of CV outcomes in an

Table 5

Univariable prediction of cardiovascular events by logistic regression analysis.

\begin{tabular}{llll}
\hline & OR & $95 \%$ CI & $p$ \\
\hline Age, years & 1.14 & $0.99-1.3$ & 0.04 \\
Males, n (\%) & 0.8 & $0.69-1.01$ & 0.9 \\
PASI & 1.2 & $1.1-1.32$ & 0.02 \\
Time elapsing since psoriasis diagnosis, years & 1.68 & $1.02-2.76$ & 0.04 \\
Presence of PsA, n (\%) & 3.13 & $1.91-19.6$ & 0.2 \\
BMI, m ${ }^{2} /$ Kg & 1.02 & $0.83-1.26$ & 0.8 \\
Hypertension, n (\%) & 24 & $2.6-34$ & 0.005 \\
Family history of heart disease, n (\%) & 1.26 & $0.13-11.7$ & 0.8 \\
Current smoking, n (\%) & 0.81 & $0.62-1.31$ & 0.9 \\
Previous smoking, n (\%) & 0.96 & $0.78-1.23$ & 0.9 \\
SBP, mmHg & 1.0 & $0.93-1.07$ & 0.9 \\
DBP, mmHg & 1.0 & $0.92-1.09$ & 0.8 \\
Total cholesterol, mg/dL & 1.01 & $0.99-1.04$ & 0.2 \\
Triglycerides, mg/dL & 1.0 & $0.98-1.02$ & 0.8 \\
hsCRP, mg/L & 1.8 & $1.3-2.14$ & 0.2 \\
hDFV, cm/s & 1.03 & $1.0-1.1$ & 0.1 \\
CFR & 7.81 & $1.93-31.2$ & 0.004 \\
\hline
\end{tabular}

Abbreviations as in Tables 1 and 4 . 
approximately log-linear manner [35,36]. IL-6 is able to promote differentiation of naive Th cells into Th17 cells, that represent the most important pathogenic cell in psoriasis lesions development [37]. In the last years, inhibitors of IL-17 and TNF- $\alpha$ have become available in the treatment of psoriasis and other inflammatory diseases. Non-randomized data from biological registries suggest that TNF- $\alpha$ blocking therapy could reduce $\mathrm{CV}$ risk in patients affected by rheumatological and dermatological diseases [38].

We have recently reported that CMD improves after treatment with TNF- $\alpha$ inhibitors in patients with established psoriasis [9]. Therefore, it is plausible that chronic inflammation represents the linking mechanism between psoriasis, CMD and increased CV risk, and that antiinflammatory treatment may help to reduce this risk.

The results of the present study have a potential impact in the management of patients with psoriasis. Cardiovascular risk stratification of patients with psoriasis remains a difficult clinical problem. Since psoriasis patients face an increased risk of coronary artery disease, it is important to identify patients at higher risk for developing cardiovascular complications in order to reduce morbidity and mortality. Screening by noninvasive Doppler-derived CFR might represent a valuable, safe and inexpensive tool for assessing the presence and prognostic effect of macro- and microvascular disease in patients with psoriasis. In our opinion no further cardiological deepening might be justified for patients with CFR $>2.5$, because of their low risk for future cardiac events. On the contrary, strict follow-up should be considered for patients with $\mathrm{CFR} \leq 2.5$. In these higher risk patients, an aggressive strategy, including the careful management of conventional CV risk factors, seems to represent the logical approach. Optimizing cardiovascular prevention and care in this patient population should involve close liaison between cardiologists and dermatologists. Furthermore, the control of psoriasis and its related systemic inflammation using powerful drugs blocking major drivers of skin and vascular inflammation (namely TNF- $\alpha$ and IL-17), may eventually impart benefits toward overall CV risk. Recently, CANTOS study showed that IL-1 inhibition by Canakinumab inhibition given once every three months among patients with a prior myocardial infarction and a persistent increase in C-reactive protein reduced the hazard ratio for cardiovascular events of $15 \%$ compared with placebo [4].

Further studies need to provide more insights on the duration of improvement of CMD obtained with appropriate psoriasis therapy during time and the real impact of a recover of blunted CFR on future cardiovascular outcomes in these patients.

As outlined above, the mechanism of atherogenesis as well as the coronary microvascular dysfunction is mainly considered an inflammatory process. Supporting this view, many studies have shown that also a low-grade systemic inflammation leads to an increased risk of cardiovascular events. Diet is one of the most important factors that can affect the inflammatory process and oxidative stress, and it could actually represent a good and relatively easy point of intervention to reduce the risk of cardiovascular disease. Indeed, some foods may have a proinflammatory effect and increase oxidative stress, while others may have a beneficial effect [39]. For example, the Western diet, mainly characterized by meat consumption, is positively associated with inflammatory biomarkers, while the Mediterranean diet has been shown to reduce some inflammatory biomarkers. Several studies have evaluated the role of individual nutrients on psoriasis [40]. In particular, the greater adherence to the Mediterranean diet is associated with a lesser severity of psoriasis, both objective and subjective, independently of the modalty of treatment and the presence of obesity, and lower levels of C-reactive protein [41]. In this context, we are not able to exclude the possible effect of the diet on the microcirculatory function in our patients. However, this influence seems unlikely as our patients' diet did not differ significantly and our patients also came largely from the same geographical area and not from areas with different eating habits.

Some important limitations need to be taken into account in the present study. It is a retrospective study, with a small sample size and with few events. The small sample size and few events did not allow us to perform a survival multivariable analysis and mostly error and nonreproducible may also occur. Even though caution must be applied while extrapolating our results, we believe our sample might be representative of the region since our medical center is a high volume reference for psoriasis and CFR assessment. There may be an inherent selection bias because of its nonrandomized design. A large-scale randomized controlled trial would be ideal. Further larger trials with larger patient populations should be performed to confirm our data and, more importantly, to establish the potential beneficial role of reversing CMD by interventional strategies for long-term atherosclerotic disease progression.

\subsection{Clinical perspective}

Our findings confirm a novel role of coronary microcirculation in the pathophysiology of cardiovascular disease in severe psoriasis. CFR by TDE may be a noninvasive, simple, and objective diagnostic tool for the assessment and follow-up of coronary microvascular function in these patients. Moreover, our data may improve the management and treatment of psoriasis in its cardiac asymptomatic initial phase and may explain why the disease is associated with increased cardiovascular morbidity and risk, although molecular mechanisms involved need to be further investigated.

\subsection{Conclusions}

The results of the present study provide evidence that CMD is associated with a greater severity of psoriasis and the presence of psoriatic arthritis, both increasing the burden of inflammation, and hypertension. Moreover, our study suggests that psoriasis patients with CMD carry a higher risk of cardiovascular events over a long-term follow up. These results not only provide a rationale for using CFR determination for cardiovascular risk assessment in psoriasis patients, but also reinforce the concept of systemic inflammation as a prognostic parameter for CMD and, eventually, for adverse long-term CV outcome. Whether novel biologic therapies able to reduce skin disease will improve CMD and prognosis in these patients needs to be further studied, prospectively.

\section{Conflicts of interest}

The authors declared they do not have anything to disclose regarding conflict of interest with respect to this manuscript.

\section{Financial support}

Dr. Osto is recipient of the Swiss National Science Foundation PRIMA Grant (PR00P3_179861/1) and of the Swiss Card-Onco-Grant (Alfred and Annemarie von Sick Grant) at the Institute of Clinical Chemistry, University Hospital Zurich, Zurich, Switzerland.

\section{Author contributions}

Stefano Piaserico: conceived of the presented idea, wrote the manuscript.

Elena Osto: conceived of the presented idea, wrote the manuscript.

Giula Famoso: Performed the CFR and contibuted to the analysis of the results.

Roberta Montisci: performed the statistical analysis.

Laura De Michieli: Performed the CFR and contibuted to the analysis of the results.

Irene Zanetti: collected the data.

Sabino Iliceto: supervised the project.

Francesco Tona: conceived of the presented idea, designed and 


\section{directed the project.}

\section{Appendix A. Supplementary data}

Supplementary data to this article can be found online at https:// doi.org/10.1016/j.atherosclerosis.2019.08.009.

\section{References}

[1] A. Faccini, J.C. Kaski, P.G. Camici, Coronary microvascular dysfunction in chronic inflammatory rheumatoid diseases, Eur. Heart J. 37 (23) (2016) 1799-1806.

[2] J.M. Gelfand, A.L. Neimann, D.B. Shin, X. Wang, D.J. Margolis, et al., Risk of myocardial infarction in patients with psoriasis, J. Am. Med. Assoc. 296 (14) (2006) 1735-1741.

[3] J.B. Lerman, A.A. Joshi, A. Chaturvedi, T.M. Aberra, A.K. Dey, et al., Coronary plaque characterization in psoriasis reveals high-risk features that improve after treatment in a prospective observational study, Circulation 136 (3) (2017) 263-276.

[4] P.M. Ridker, B.M. Everett, T. Thuren, J.G. MacFadyen, W.H. Chang, et al., CANTOS trial group. Antiinflammatory therapy with Canakinumab for atherosclerotic disease, N. Engl. J. Med. 377 (12) (2017) 1119-1131.

[5] Y.A. Elnabawi, A.K. Dey, A. Goyal, J.W. Groenendyk, J.H. Chung, et al., Coronary artery plaque characteristics and treatment with biologic therapy in severe psoriasis: results from a prospective observational study, Cardiovasc. Res. 115 (2019) 721-728.

[6] J.J. Wu, K.-Y.T. Poon, J.C. Channual, A.Y.-J. Shen, Association between tumor necrosis factor inhibitor therapy and myocardial infarction risk in patients with psoriasis, Arch. Dermatol. 148 (2012) 1244-1250.

[7] P.G. Camici, F. Crea, Coronary microvascular dysfunction, N. Engl. J. Med. 356 (2007) 830-840.

[8] E. Osto, S. Piaserico, A. Maddalozzo, G. Forchetti, R. Montisci, et al., Impaired coronary flow reserve in young patients affected by severe psoriasis, Atherosclerosis 221 (1) (2012) 113-117.

[9] S. Piaserico, E. Osto, G. Famoso, I. Zanetti, D. Gregori, et al., Treatment with tumor necrosis factor inhibitors restores coronary microvascular function in young patients with severe psoriasis, Atherosclerosis 251 (2016) 25-30.

[10] A. Vacca, R. Montisci, P. Garau, P. Siotto, M. Piga, et al., Prognostic impact of coronary microcirculation abnormalities in systemic sclerosis: a prospective study to evaluate the role of noninvasive tests, Arthritis Res. Ther. 15 (2013) R8.

[11] R.B. Devereux, D.R. Alonso, E.M. Lutas, G.J. Gottlieb, E. Campo, et al., Echocardiographic assessment of left ventricular hypertrophy: comparison to necropsy findings, Am. J. Cardiol. 57 (1986) 450-458.

[12] S.F. Nagueh, C.P. Appleton, T.C. Gillebert, P.N. Marino, J.K. Oh, et al., Recommendations for the evaluation of left ventricular diastolic function by echocardiography, J. Am. Soc. Echocardiogr. 22 (2009) 107-133.

[13] M.M. Redfield, S.J. Jacobsen, J.C. Burnett Jr., D.W. Mahoney, K.R. Balley, et al., Burden of systolic and diastolic ventricular dysfunction in the community: appreciating the scope of the heart failure epidemic, J. Am. Med. Assoc. 289 (2003) 194-202.

[14] F. Tona, A.L.P. Caforio, R. Montisci, A. Gambino, A. Angelini, et al., Coronary flow velocity pattern and coronary flow reserve by contrast-enhanced transthoracic echocardiography predict long-term outcome in heart transplantation, Circulation 114 (Suppl. I) (2006) I-49-55.

[15] R. Rubinshtein, E.H. Yang, C.S. Rihal, A. Prasad, R.J. Lennon, et al., Coronary microcirculatory vasodilator function in relation to risk factors among patients without obstructive coronary disease and low to intermediate Framingham score, Eur. Heart J. 31 (2009) 936-942.

[16] V. Russo, A. Zavalloni, M.L. Bacci Reggiani, K. Buttazzi, V. Gostoli, et al., Incremental prognostic value of coronary CT angiography in patients with suspected coronary artery disease, Circ. Cardiovasc. Imaging 3 (2010) 351-359.

[17] K. Thygesen, J.S. Alpert, A.S. Jaffe, B.R. Chaitman, J.J. Bax, et al., Executive group on behalf of the joint european society of cardiology (ESC)/American college of cardiology (ACC)/American heart association (AHA)/World heart federation (WHF) task force for the universal definition of myocardial infarction, J. Am. Coll. Cardiol. 18 (2018) 2231-2264.

[18] M. Roffi, C. Patrono, J.P. Collet, C. Mueller, M. Valgimigli, et al., ESC Scientific Document Group, 2015 ESC guidelines for the management of acute coronary syndromes in patients presenting without persistent ST-segment elevation: task force for the management of acute coronary syndromes in patients presenting without persistent ST-segment elevation of the european society of cardiology
(ESC), Eur. Heart J. 37 (2016) 267-315.

[19] J.E. Tamis-Holland, H. Jneid, Myocardial Infarction with nonobstructive coronary arteries (MINOCA): it's time to face reality!, J. Am. Heart. Assoc. 7 (13) (2018) pii: e009635.

[20] N.N. Mehta, R.S. Azfar, D.B. Shin, A.L. Neimann, A.B. Troxel, et al., Patients with severe psoriasis are at increased risk of cardiovascular mortality: cohort study using the general practice research database, Eur. Heart J. 31 (2010) 1000-1006.

[21] J.M. Gelfand, A.B. Troxel, J.D. Lewis, S.K. Kurd, D.B. Shin, et al., The risk of mortality in patients with psoriasis: results from a population-based study, Arch. Dermatol. 143 (2007) 1493-1499.

[22] Y.B. Brauchli, S.S. Jick, M. Miret, C.R. Meier, Psoriasis and risk of incident myocardial infarction, stroke or transient ischaemic attack: an inception cohort study with a nested case-control analysis, Br. J. Dermatol. 160 (2009) 1048-1056.

[23] N.N. Mehta, Y. Yu, R. Pinnelas, P. Krishnamoorthy, D.B. Shin, et al., Attributable risk estimate of severe psoriasis on major cardiovascular events, Am. J. Med. 124 (2011) 775e1-775e6.

[24] R. Sicari, F. Rigo, L. Cortigiani, S. Gherardi, M. Galderisi, et al., Additive prognostic value of coronary flow reserve in patients with chest pain syndrome and normal or near-normal coronary arteries, Am. J. Cardiol. 103 (2009) 626-631.

[25] K. Nakanishi, S. Fukuda, K. Shimada, C. Miyazaki, K. Otsuka, et al., Impaired coronary flow reserve as a marker of microvascular dysfunction to predict long-term cardiovascular outcomes, acute coronary syndrome and the development of heart failure, Circ. J. 76 (2012) 1958-1964.

[26] L. Cortigiani, F. Rigo, S. Gherardi, M. Galderisi, F. Bovenzi, et al., Prognostic meaning of coronary microvascular disease in type 2 diabetes mellitus: a transthoracic Doppler echocardiographic study, J. Am. Soc. Echocardiogr. 27 (7) (2014) $742-748$.

[27] T. Kawata, M. Daimon, R. Hasegawa, T. Toyoda, T. Sekine, et al., Prognostic value of coronary flow reserve assessed by transthoracic Doppler echocardiography on long-term outcome in asymptomatic patients with type 2 diabetes without overt coronary artery disease, Cardiovasc. Diabetol. 12 (2013) 121-128.

[28] R. Arroyo-Espliguero, N. Mollichelli, P. Avanzas, E. Zouridakis, V.R. Newey, et al., Chronic inflammation and increased arterial stiffness in patients with cardiac syndrome X, Eur. Heart J. 24 (2003) 2006-2011.

[29] M. Caliskan, D. Erdogan, H. Gullu, S. Yilmaz, Y. Gursoy, et al., Impaired coronary microvascular and left ventricular diastolic functions in patients with ankylosing spondylitis, Atherosclerosis 196 (2008) 306-312.

[30] A. Recio-Mayoral, J.C. Mason, J.C. Kaski, M.B. Rubens, O.A. Harari, et al., Chronic inflammation and coronary microvascular dysfunction in patients without risk factors for coronary artery disease, Eur. Heart J. 30 (2009) 1837-1843.

[31] P.A. Kaufmann, T. Gnecchi-Ruscone, T.M. Di, K.P. Schafers, T.F. Luscher, et al. Coronary heart disease in smokers: vitamin C restores coronary microcirculatory function, Circulation 102 (2000) 1233-1238.

[32] A. Asahina, Y. Umezawa, K. Yanaba, H. Nakagawa, Serum C-reactive protein levels in Japanese patients with psoriasis and psoriatic arthritis: long-term differential effects of biologics, J. Dermatol. 43 (7) (2016 Jul) 779-784.

[33] I. Snekvik, T.I.L. Nilsen, P.R. Romundstad, M. Saunes, Psoriasis and cardiovascular disease risk factors: the HUNT Study, Norway, J. Eur. Acad. Dermatol. Venereol. 32 (5) (2018 May) 776-782.

[34] H. Zhang, Y. Park, J. Wu, Xp Chen, S. Lee, et al., Role of TNF-alpha in vascular dysfunction, Clin. Sci. 116 (3) (2009) 219-230.

[35] M. Woodward, P. Welsh, A. Rumley, H. Tunstall-Pedoe, G.D. Lowe, Do in flammatory biomarkers add to the discrimination of cardiovascular disease after allowing for social deprivation? Results from a 10-year cohort study in Glasgow, Scotland, Eur. Heart J. 31 (21) (2010 Nov) 2669-2675.

[36] S. Kaptoge, S.R. Seshasai, P. Gao, D.F. Freitag, A.S. Butterworth, et al., Inflammatory cytokines and risk of coronary heart disease: new prospective study and updated meta-analysis, Eur. Heart J. 35 (9) (2014 Mar) 578-589.

[37] A. Beringer, M. Noack, P. Miossec, IL-17 in Chronic Inflammation: from discovery to targeting, Trends Mol. Med. 22 (3) (2016) 230-241.

[38] L. Puig, Cardiometabolic comorbidities in psoriasis and psoriatic arthritis, Int. J. Mol. Sci. 25 (19) (2017) 58-74.

[39] A.M. Minihane, S. Vinoy, W.R. Russell, A. Baka, H.M. Roche, et al., Low-grade in flammation, diet composition and health: current research evidence and its translation, Br. J. Nutr. 114 (2015) 999-1012.

[40] A. Molina-Leyva, C. Cuenca-Barrales, J.J. Vega-Castillo, J.C. Ruiz-Carrascosa, R. Ruiz-Villaverde, Adherence to mediterranean diet in Spanish patients with psoriasis: cardiovascular benefits? Dermatol. Ther. 32 (2019) e12810.

[41] C. Phan, M. Touvier, E. Kesse-Guyot, M. Adjibade, S. Hercberg, et al., Association between mediterranean anti-inflammatory dietary profile and severity of psoriasis: results from the NutriNet-Santé Cohort, JAMA Dermatol. 154 (2018) 1017-1024. 